\begin{tabular}{|c|l|}
\hline Title & Numerical visual ization of boundary layer transition when negative Magnus effect occurs \\
\hline Author(s) & Muto, Masaya; Tsubokura, Makoto; Oshima, Nobuy uki \\
\hline Citation & Journal of Visualization, 15(3), 261-268 \\
\hline htps://doi.org/L0.1007/312650-012-0125-2 \\
\hline Issue Date & 2012-08 \\
\hline Doc URL & http://hdl.handle.net/2115/53053 \\
\hline Rights & The original publication is available at www.springerlink.com \\
\hline Type & article (author version) \\
\hline File Information & JoV 15-3_261-268.pdf \\
\hline
\end{tabular}

Instructions for use 


\title{
Numerical visualization of boundary layer transition when negative Magnus effect occurs
}

\author{
Masaya Muto \\ High Efficiency Power Generation Sector, Energy Engineering Research \\ Laboratory, Central Research Institute of Electric Power Industry, 2-6-1 \\ Nagasaka, Yokosuka, Kanagawa, 240-0196, Japan \\ Tel: +81-70-5557-7572 \\ Fax: +81-46-856-3346 \\ E-mail: masayam@criepi.denken.or.jp
}

Makoto Tsubokura

Division of Mechanical and Space Engineering, Faculty of Engineering, Hokkaido University, Kita 13, Nishi 8, Kita-ku, Sapporo, Hokkaido, 060-8628, Japan

Tel: +81-11-706-6723

Fax: +81-11-706-6723

E-mail: mtsubo@eng.hokudai.ac.jp

Nobuyuki Oshima

Division of Mechanical and Space Engineering, Faculty of Engineering, Hokkaido University, Kita 13, Nishi 8, Kita-ku, Sapporo, Hokkaido, 060-8628, Japan

Tel: +81-11-706-6722

Fax: +81-11-706-6722

E-mail: oshima@eng.hokudai.ac.jp 


\section{Abstract}

We previously identified the appearance of negative Magnus lift on a sphere rotating about an axis perpendicular to an incoming flow at a critical Reynolds number using large-eddy simulation and obtained the statistically time-averaged lift and pressure coefficients around the sphere. We have now numerically investigated the unsteady characteristics of the boundary layer around a rotating sphere at three Reynolds numbers $\left(1.0 \times 10^{4}, 2.0 \times 10^{5}\right.$, and $\left.1.14 \times 10^{6}\right)$. At a Reynolds number in the subcritical or supercritical region, the direction of the lift force followed the Magnus effect independent of the rotational speed. In contrast, at the critical Reynolds number when a particular rotational speed was imposed, negative lift was observed and a boundary-layer transition occurred only on one side of the sphere, as indicated by the visualization of the vortical structures around the sphere. A change in these structures and a shift of the separation points along with a change in the Reynolds number or rotational speed of the sphere were investigated in the context of boundary layer transition by using visualization around the sphere.

Keywords: bluff body aerodynamics, Magnus effect, boundary layer transition, large-eddy simulation

\section{Introduction}

The Magnus effect is a phenomenon by which a clockwise-rotating sphere or cylinder subjected to a left-to-right flow has a lift force [1]. While the mechanism is usually explained in terms of the inviscid flow, the same phenomenon is also used to explain the curious and unpredictable motions of the spinning balls used in such sports as baseball, volleyball, and football. The phenomenon in these cases is complicated due to viscous effects in the flow as the boundary layer on the surface of the ball separates due to an adverse pressure gradient. The difference in the relative velocity of the sphere surface with respect to the incoming uniform flow generally makes the boundary layer on the downstreammoving side (upper side in this context) thinner than that on the upstream-moving side. Thus, boundary-layer separation is delayed on the downstream-moving side while it is accelerated on the upstream-moving side. As a result, the wake flow is distorted downward, and a positive lift force, based on the principle of action and reaction, acts on the sphere. In addition, the laminar-turbulent transition of the boundary layer complicates the phenomenon even more as it causes the separation 
point to shift considerably downstream along the surface. Thus, the drag on the non-rotating sphere substantially decreases around the critical Reynolds number. Experimental studies have shown that, as a result of the interaction between these separation and transition phases, there is a negative lift force on a rotating sphere at a specific rotational speed around the critical Reynolds number [2][3][4]. This contradicts the Magnus effect. However, investigating the characteristics and spatial structure of the flow near or on a sphere is difficult due to the effect of the supporting rods or wires. This results in a quantitative disagreement between the reported magnitude of the lift force and the Reynolds number range or between the reported magnitude and the rotational speed at which the negative Magnus effect is observed. Hence, little knowledge has so far been obtained on the flow characteristics when a boundary-layer transition occurs around the critical Reynolds number.

Our objective in the study reported here was to investigate the instantaneous characteristics of the flow near the boundary layer and separation point on a sphere surface around the critical Reynolds number by using numerical simulation.

\section{Numerical method and conditions}

\section{Computational domain and boundary conditions}

The computational domain was a rectangular duct with a sphere fixed at its center, as shown in Fig. 1[5]. Thirty prism-mesh layers were placed on the sphere surface for use in resolving the velocity profile of the boundary layer. The spatial resolution around the sphere was set so as to reproduce the drag reduction at the critical Reynolds number, which was determined by the laminar boundary layer thickness $\delta_{\mathrm{B}}$ on the sphere. The thickness was estimated using the equation proposed by Schlichting [6], $\delta_{B}=3 \times \sqrt{\frac{\left(D_{p} / 2\right) v}{U_{0}}}$. The thickness of the first layer in the vicinity of the sphere surface in the radial direction was around $1 / 20 \delta_{B}$. In the circumferential direction, it was about the same as $\delta_{\mathrm{B}}$. The other region of the domain was divided by tetrahedral grids except for several prism layers on the inlet, outlet, and sidewall boundaries. An overview of the spatial resolution near 
the sphere at each Reynolds number is shown in Fig. 2. The total number of elements was about 5.3, 44, and 48 million for Reynolds numbers of $1.0 \times 10^{4}$, $2.0 \times 10^{5}$, and $1.14 \times 10^{6}$, respectively. A no-slip boundary condition was used on the sphere surface, and the clockwise rotation of the sphere was represented by the rotating velocity distribution on the surface. A uniform velocity profile without perturbation was imposed as the inlet boundary condition. The gradient-free condition for the pressure and velocity was applied to the duct outlet.

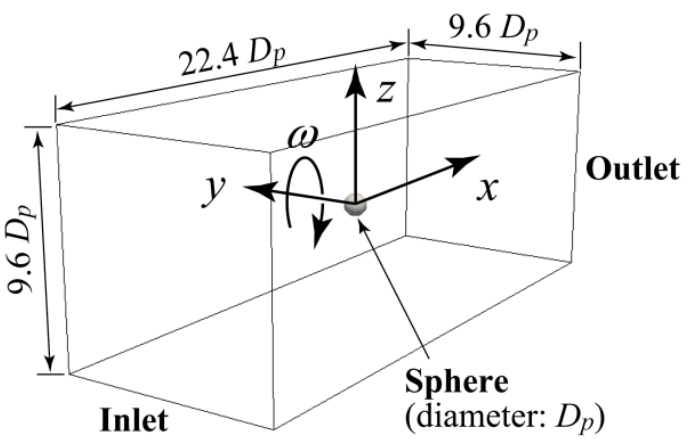

Fig. 1. Coordinate system and geometry of computational domain [5]

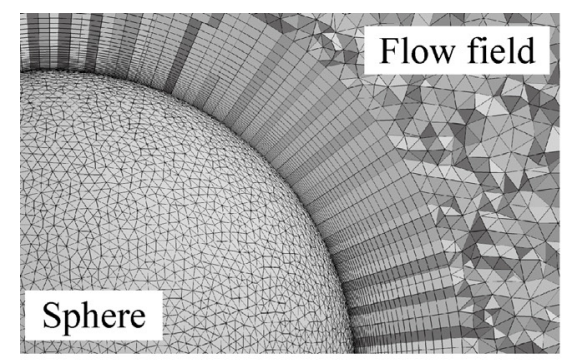

(a)

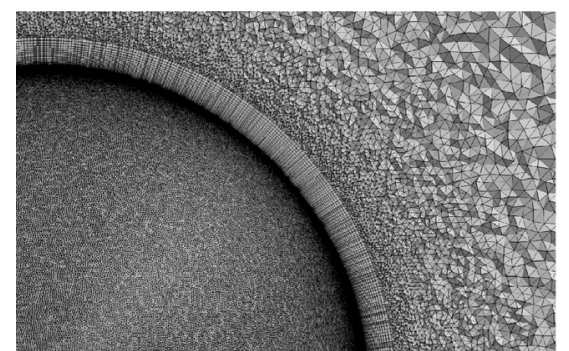

(b)

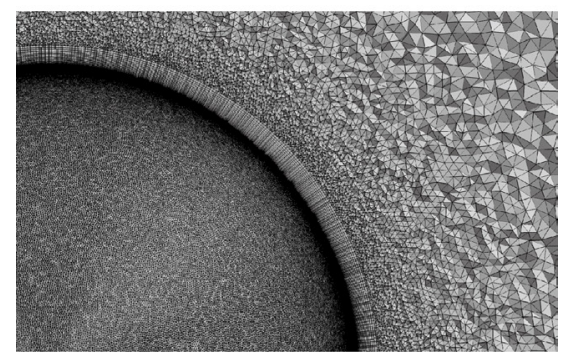

(c) 
Fig. 2. Spatial resolution of the numerical grids allocated around the sphere: (a) $R e_{p}=1.0 \times 10^{4}$; (b) $2.0 \times 10^{5}$; (c) $1.14 \times 10^{6}$

\section{Numerical conditions}

The non-dimensional rotational speed, which is conventionally called the spin parameter, is defined as the ratio of the circumferential velocity to the incoming flow velocity, $\Gamma=D_{p} \omega / U_{0}$, in which $D_{p}$ is the sphere diameter, $\omega$ is the rotational angular velocity, and $U_{0}$ is the incoming flow velocity. In previous experimental studies [2][3][4], the negative Magnus effect was observed at $\Gamma$ less than 0.5 . Thus, we considered two non-dimensional velocities of $\Gamma=0.2$ and 1 , and Reynolds numbers $R e_{p}=U_{0} D_{p} / v$ ( $v$ is kinetic viscosity) of $1.0 \times 10^{4}, 2.0 \times 10^{5}$, and $1.14 \times 10^{6}$ in the subcritical, critical, and supercritical flow ranges [7], respectively. To investigate the high-Reynolds-number turbulent flow including transition, we used large-eddy simulation (LES) with a dynamic subgrid-scale model as the numerical method.

Figure 3 plots the drag coefficient profiles obtained for each Reynolds number condition; previously obtained experimental data [6][7][8] are shown for reference. Two additional Reynolds number conditions $\left(1.6 \times 10^{5}\right.$ and $\left.2.4 \times 10^{5}\right)$ were used to determine whether our numerical method correctly reproduces the drag crisis in the critical flow regime. The drag coefficient is defined as $C_{D}=\frac{F_{D}}{\frac{1}{2} \rho U_{0}^{2} A}$, where $F_{D}, \rho$, and $A$ are the drag force, fluid density, and projected area of the sphere, respectively. The drag reduction, in which the drag substantially decreases as the Reynolds number increases, was noticeable from $1 \times 10^{5}$ to $3 \times 10^{5}$. The drag crisis near the critical Reynolds number was thus accurately captured with this method.

The variation in the lift coefficient with respect to the spin parameter is plotted in Fig. 4. For reference, along with reported data [3][4][9] for reference. The lift coefficients at $\Gamma=0.1,0.5$, and 0.8 are also plotted to clarify the trend against the spin parameter in the critical flow regime. The lift coefficient is defined as $C_{L}=\frac{F_{L}}{\frac{1}{2} \rho U_{0}^{2} A}$, where $F_{L}$ is the aerodynamic lift force acting in the $y$-direction. Our numerical results for $\operatorname{Re}_{p}=1.0 \times 10^{4}$ in the subcritical flow regime accurately reproduced the trend reported by Tsuji et al. Both the numerical and experimental 
results indicate that the conventional Magnus effect induces a positive lift force on the sphere. On the other hand, the two experimental trends near the critical flow regime suggest the existence of negative lift at relatively slower rotational speeds between $\Gamma$ of 0.1 and 0.5 . The results of our LES for $R e_{p}=2.0 \times 10^{5}$ and $\Gamma=0.2$ confirm the existence of the negative Magnus lift. At the higher rotational speed for the supercritical flow regime, the trend of the lift coefficient was the same as that for the subcritical regime, suggesting that negative Magnus lift does not appear.

We ran 256 parallel CPU simulations with approximately 30,000 time steps (20,000 steps for initial transients; 10,000 for statistics). The calculation time required for each case was approximately 60 hours on a Hitachi SR11000 supercomputer.

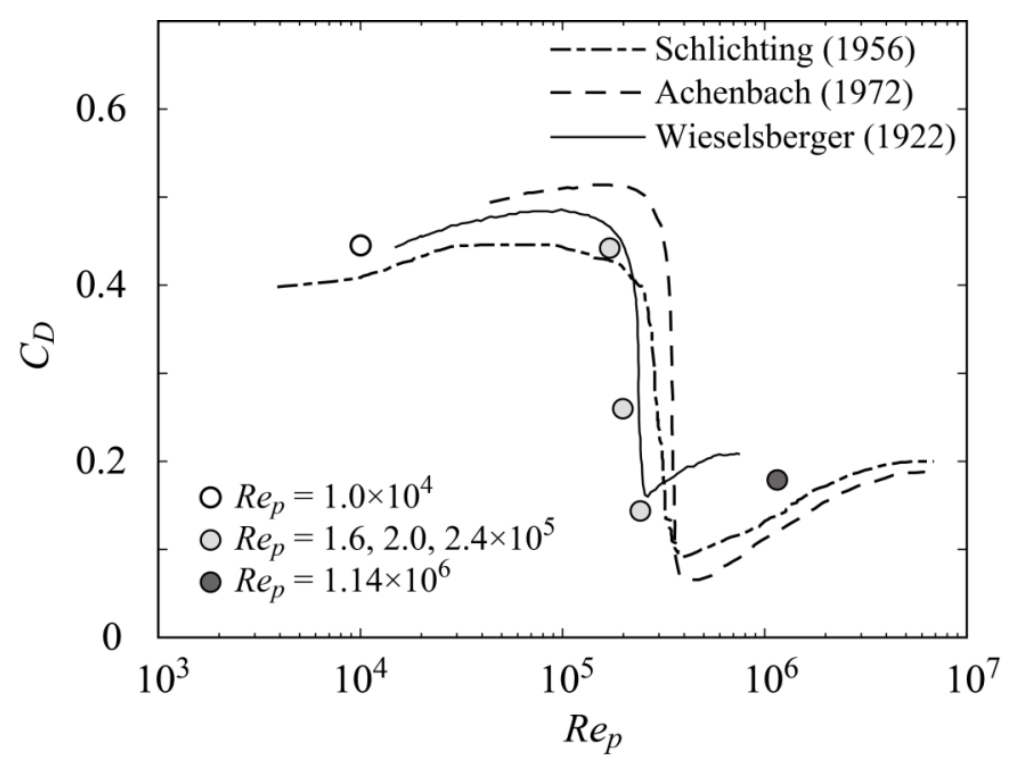

Fig. 3. Drag coefficient as function of Reynolds number in subcritical, critical, and supercritical flow regimes $(\Gamma=0)[5]$ 


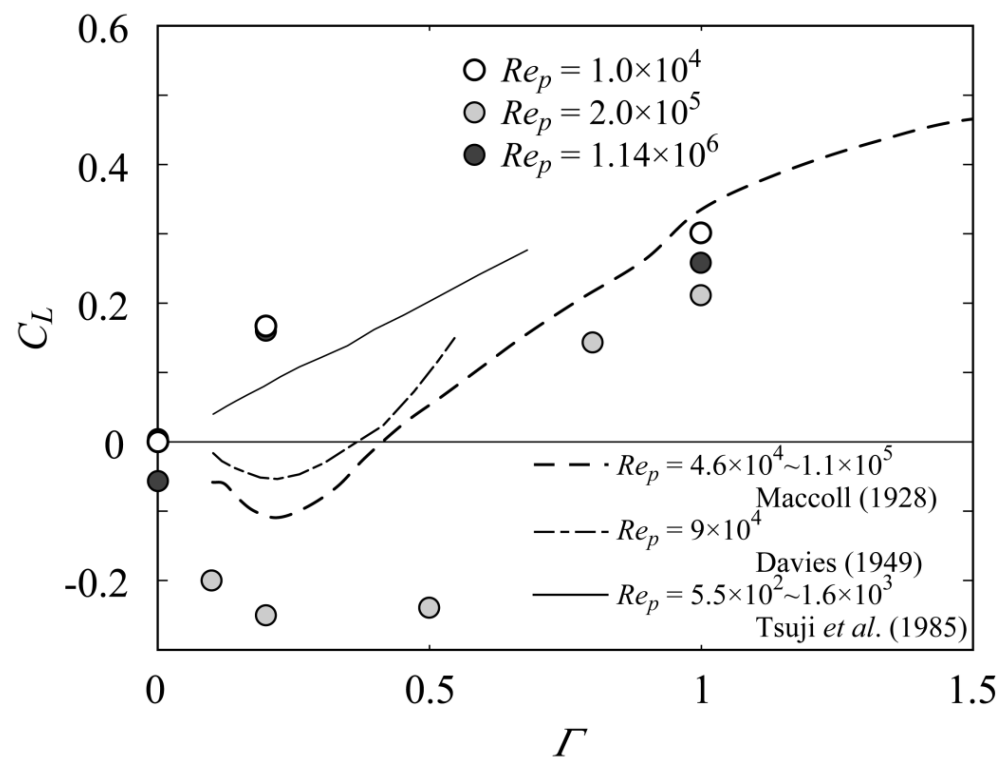

Fig. 4. Variation in lift coefficient with respect to spin parameter [5] 


\section{Visualization of boundary-layer separation}

\section{Change in vortex structure by rotation}

Figures 5(a) to (i) show images of the instantaneous vortical structures around a non-rotating or rotating sphere at each Reynolds number. The obtained structures were an iso-surface of the second invariant of the velocity gradient tensor. For both the non-rotating and rotating spheres at $R e_{p}=1.0 \times 10^{4}$, as shown in Figs. 5(a) to (c), we could not identify any noticeable structural vortex on the sphere surface we could identify one for the other Reynolds numbers. At $R e_{p}=2.0 \times 10^{5}$ for a non-rotating sphere (Fig. 5(d)), azimuthal tube-like vortex structures partially covered the sphere surface, indicating that a boundary layer transition had occurred. Tube-like vortex structures were not identified around the first separation point, suggesting that the separation is in the laminar state. In a previous study, the first separation point of the boundary layer was identified as the streamwise time-averaged velocity of the first nearest grid point from where the sphere surface becomes negative [5]. In the same way, the reattachment point was identified as the velocity becomes positive again. According to this definition, the separation point was located at $\phi=87^{\circ}, 92^{\circ}$, and $103^{\circ}$ at $R e_{p}=$ $1.0 \times 10^{4}, 2.0 \times 10^{5}$, and $1.14 \times 10^{6}$, respectively. The reattachment and re-separation of the boundary layer were observed at $98^{\circ}$ and $104^{\circ}$, respectively, at $R e_{p}=$ $2.0 \times 10^{5}$. The $\phi$ is the polar angle from the frontal stagnation point.

The transition of the boundary layer occurs around the reattachment point. When the sphere rotates at $\Gamma=0.2$, a negative Magnus effect appears, as indicated by the pressure distribution on the upstream-moving side on the sphere surface becoming lower than that on the downstream-moving side (Fig. 6). The boundary layer on the upstream-moving side is perturbed around the first separation point. This perturbation apparently makes the laminar separation and reattachment points indistinct. For both the non-rotating and rotating spheres at $R e_{p}=1.14 \times 10^{6}$ (Figs. $5(\mathrm{~g})$ to (i)), vortex structures covered the sphere surface from around the first separation point, suggesting that the boundary layer had sufficiently transitioned to the turbulent state. 


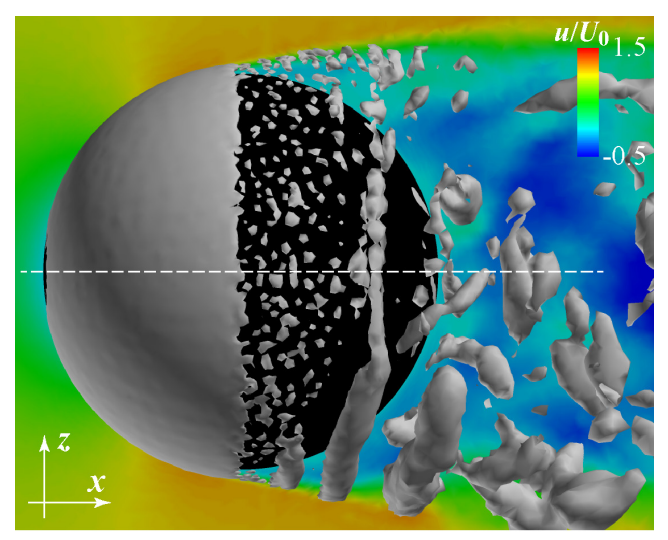

(a) $R e_{p}=1.0 \times 10^{4}, \Gamma=0$

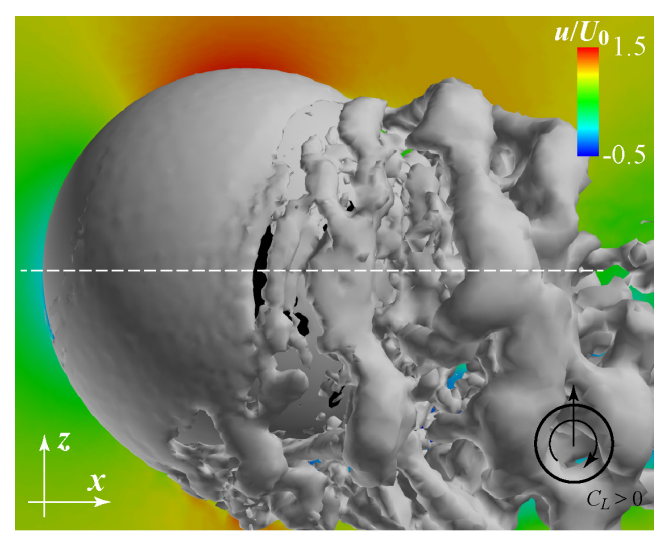

(c) $R e_{p}=1.0 \times 10^{4}, \Gamma=1.0$

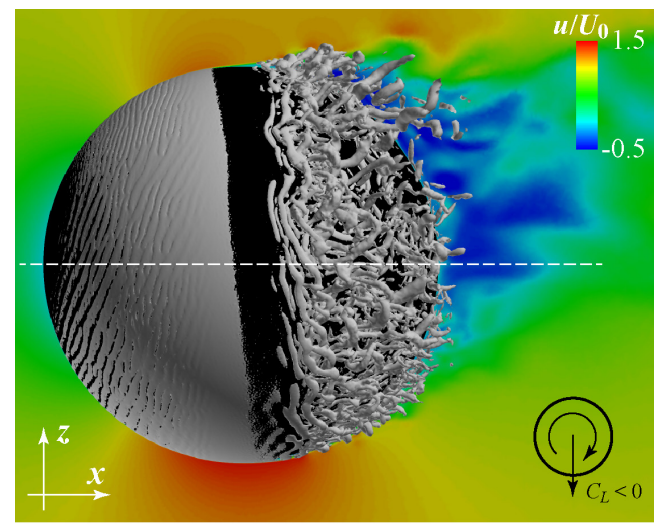

(e) $R e_{p}=2.0 \times 10^{5}, \Gamma=0.2$

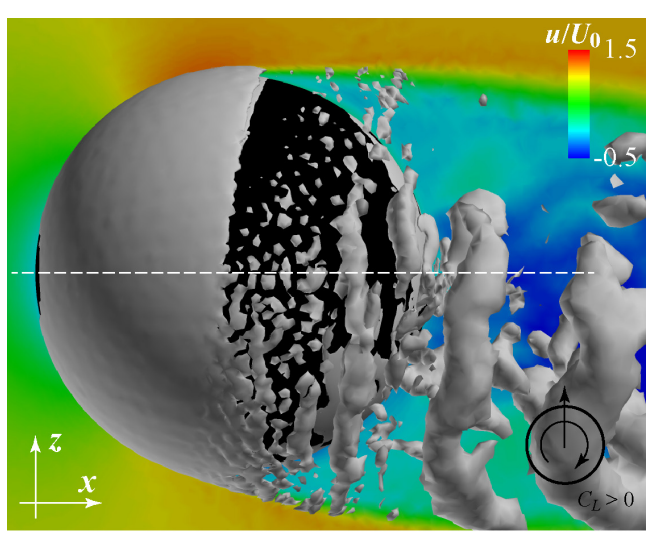

(b) $R e_{p}=1.0 \times 10^{4}, \Gamma=0.2$

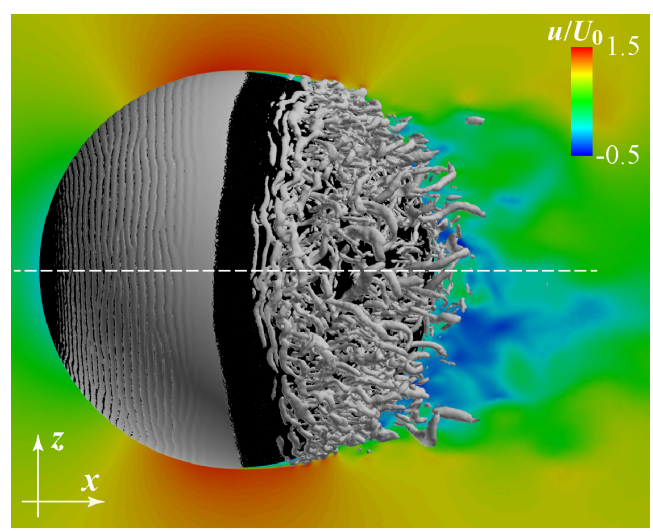

(d) $R e_{p}=2.0 \times 10^{5}, \Gamma=0$

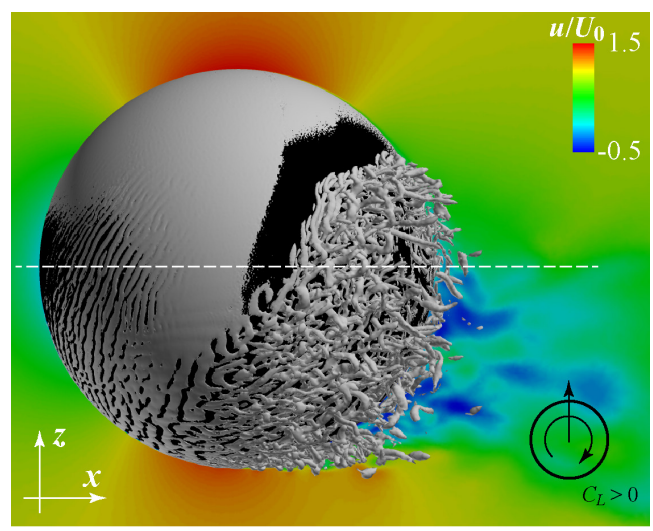

(f) $R e_{p}=2.0 \times 10^{5}, \Gamma=1.0$ 


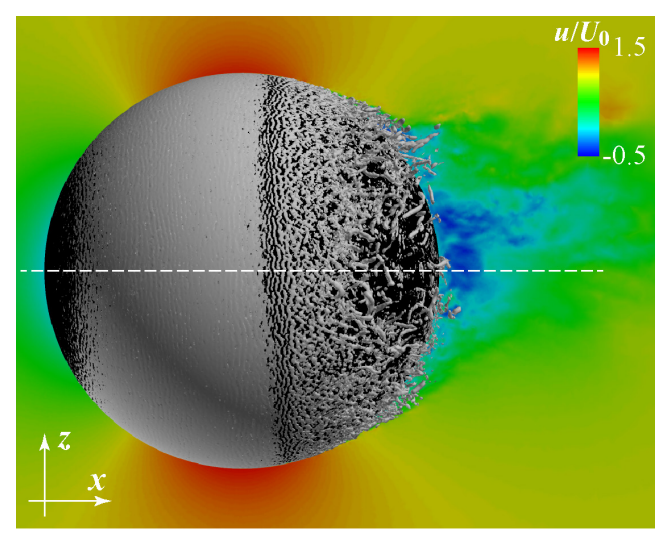

(g) $R e_{p}=1.14 \times 10^{6}, \Gamma=0$

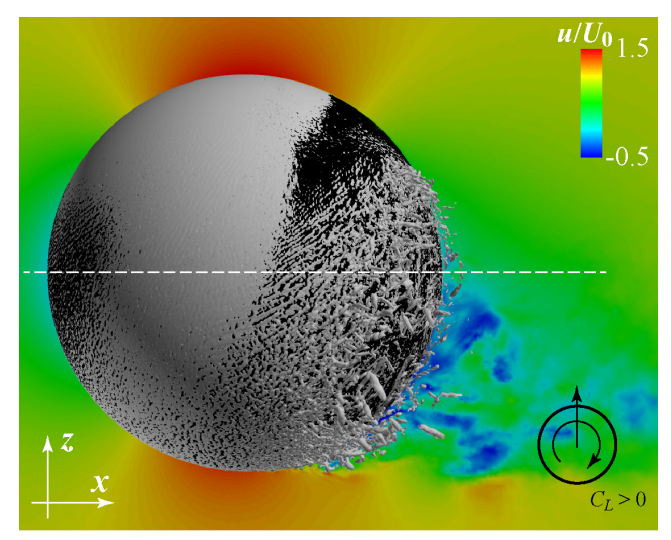

(i) $R e_{p}=1.14 \times 10^{6}, \Gamma=1.0$

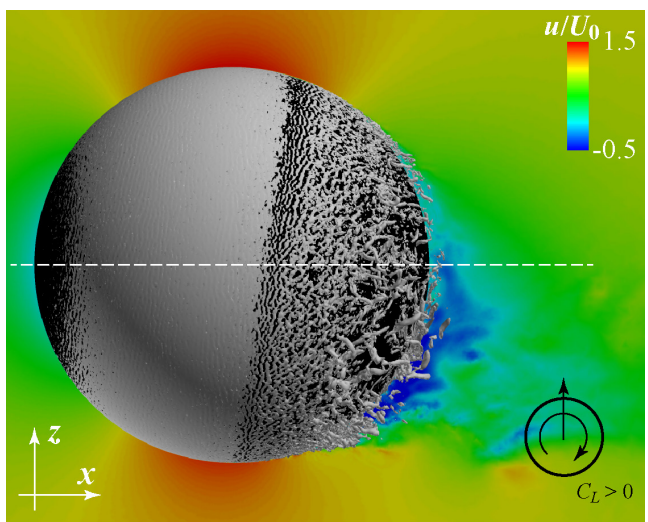

(h) $\operatorname{Re}_{p}=1.14 \times 10^{6}, \Gamma=0.2$

Fig. 5. Contours of streamwise velocity ( $x$ component) on central cross section $(y=0)$ with instantaneous vortical structures around sphere (iso-surface of second invariant of velocity gradient tensor) under various conditions

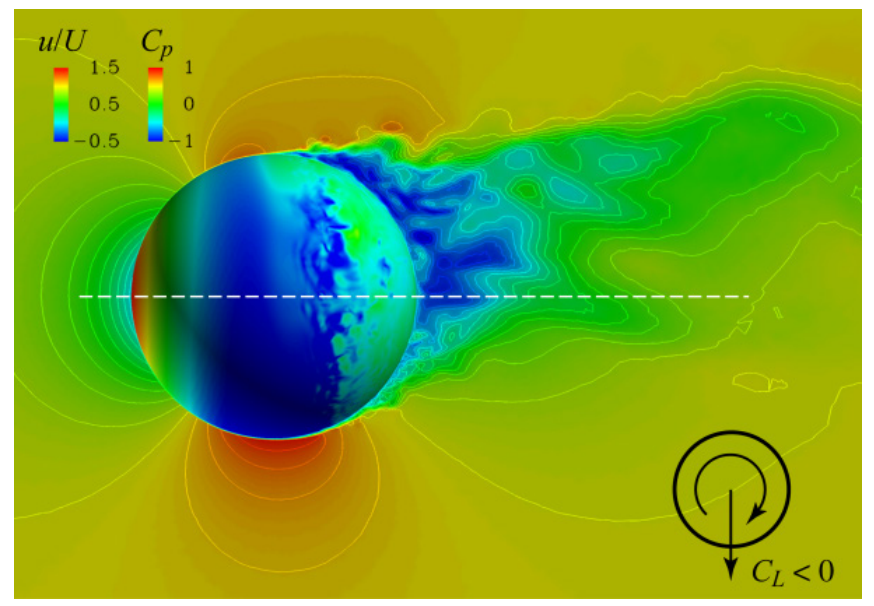

Fig. 6. Instantaneous contours of streamwise velocity ( $x$ component) on central cross section $(y=$ 0 ), and static pressure coefficient on sphere surface at $\operatorname{Re}_{p}=2.0 \times 10^{5}$ and $\Gamma=0.2$ [5] 


\section{Spatial distribution of separation points on sphere surface}

The distribution of the separation points on the sphere surface changes when the boundary layer becomes turbulent. Figures 7(a) to (i) show the instantaneous isoline of the zero-shear stress on the surface of non-rotating and rotating spheres at each Reynolds number. The coordinate system used for these figures is shown in Fig. 8.

For $R e_{p}=1.0 \times 10^{4}$ (Figs. 7(a) to (c)), the iso-line is distributed smoothly compared to that for the other Reynolds numbers. As the rotational speed increased, the separation line shifted along with the rotation of the sphere on the downwardmoving side (Figs. 7(b) and (c)) while the separation line on the upward-moving side became invisible. The reason for this was apparently due to the rotational speed of the sphere exceeding the counter flow velocity generated along with the separation for the non-rotational sphere. Subsequently, the point where the relative velocity between the sphere surface and the flow near the sphere became zero disappeared.

For $R e_{p}=2.0 \times 10^{5}$, the distribution of the iso-line for the rotating sphere (Fig. 7(e)) shows that, with a rotating sphere, the separation points form small discrete regions on the upstream-moving side, which indicates that boundary-layer transition occurs on that side and that the velocity distribution is more perturbed than that for a non-rotating sphere, as shown in Fig. 7(d). When the rotational speed was 1 (Fig. 7(f)), the distribution of separation points became smooth because the relative velocity between the sphere surface and streamwise velocity approached zero.

Finally, for $R e_{p}=1.14 \times 10^{6}$ (Figs. 7 (g) to (i)), the distribution of separation points was not smooth. The points formed small regions on both the upstream and downstream-moving sides of the sphere regardless of the rotation speed. This is because the boundary layer on the sphere fully transited to the turbulent state. 


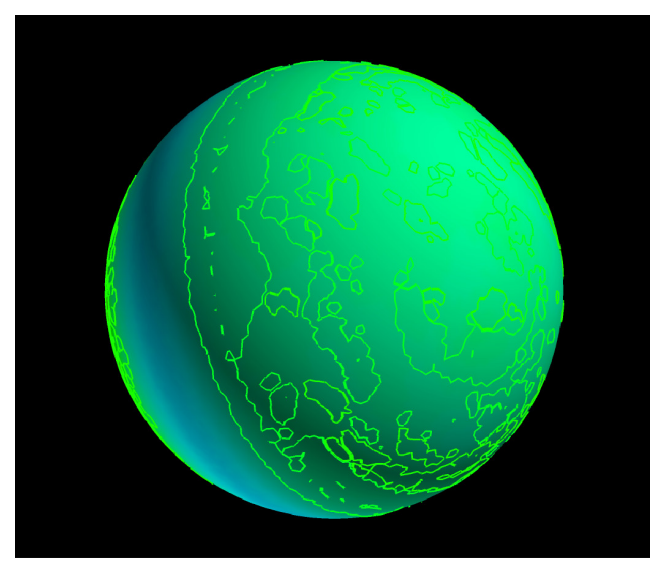

(a) $R e_{p}=1.0 \times 10^{4}, \Gamma=0$

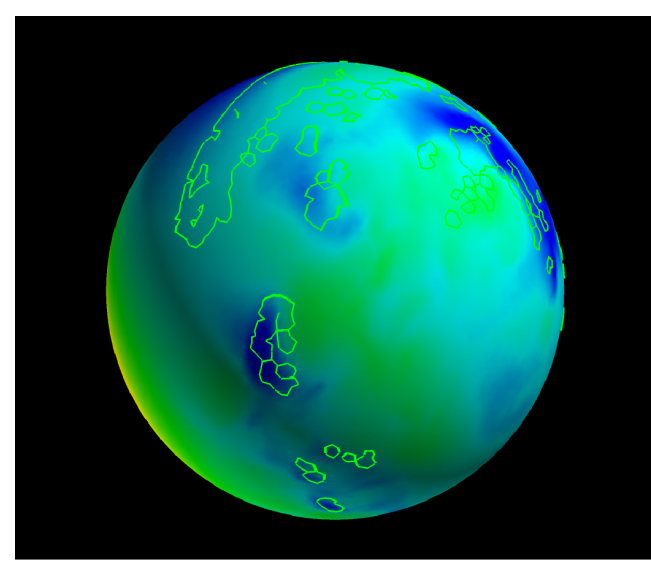

(c) $R e_{p}=1.0 \times 10^{4}, \Gamma=1.0$

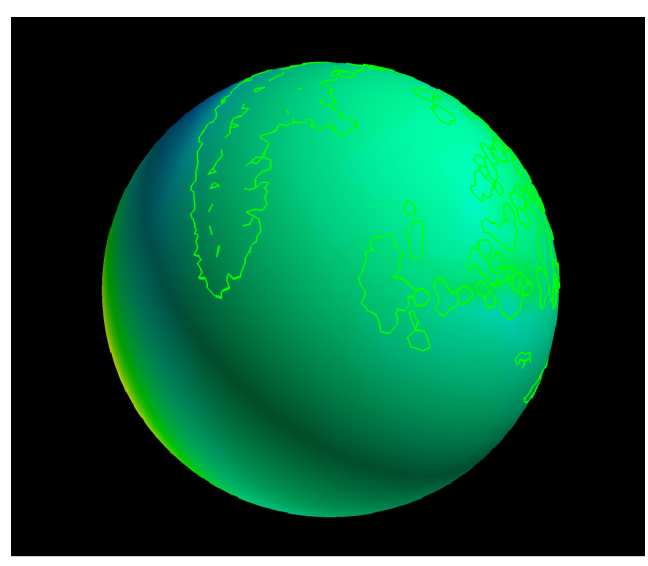

(b) $R e_{p}=1.0 \times 10^{4}, \Gamma=0.2$

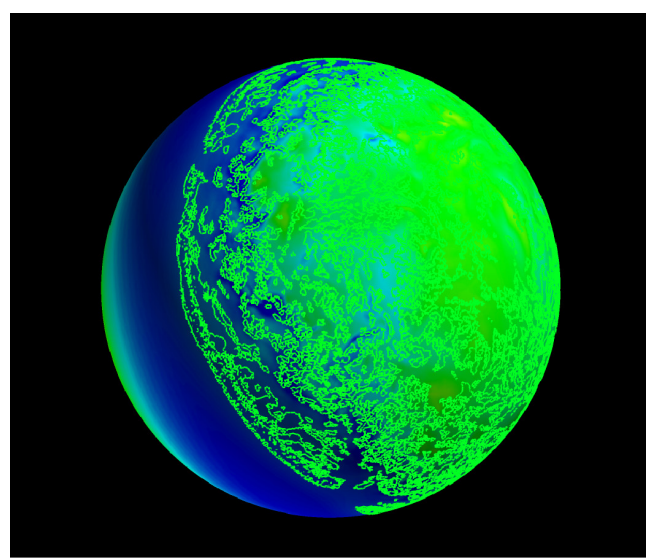

(d) $R e_{p}=2.0 \times 10^{5}, \Gamma=0$

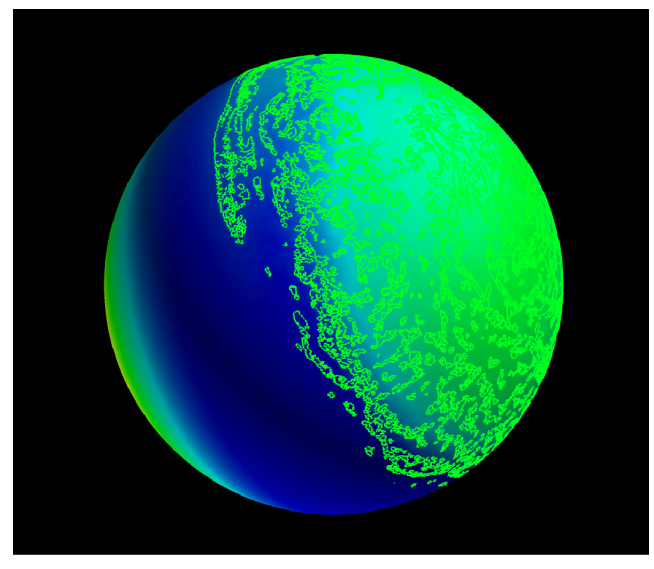

(e) $R e_{p}=2.0 \times 10^{5}, \Gamma=0.2$

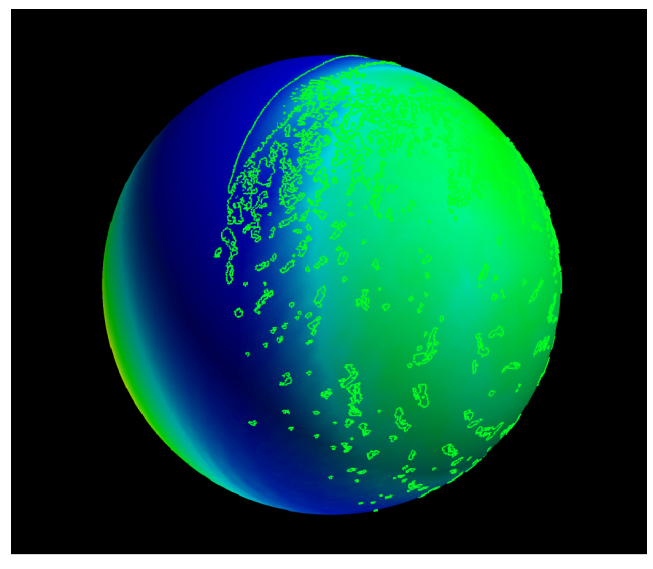

(f) $R e_{p}=2.0 \times 10^{5}, \quad \Gamma=1.0$ 


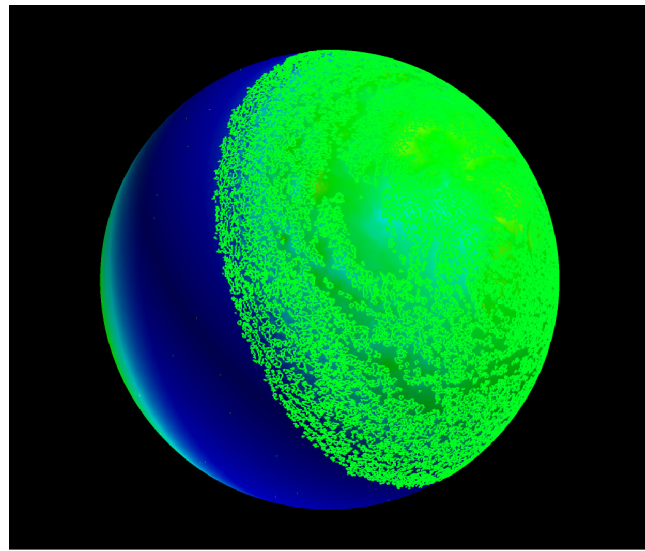

(g) $R e_{p}=1.14 \times 10^{6}, \Gamma=0$

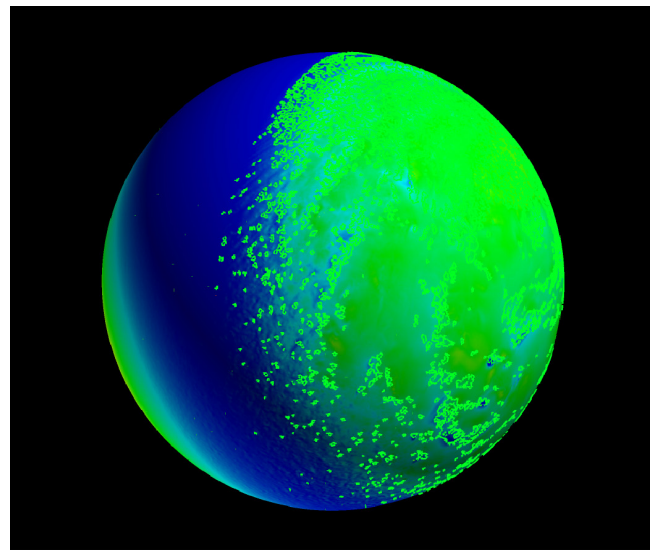

(i) $R e_{p}=1.14 \times 10^{6}, \Gamma=1.0$

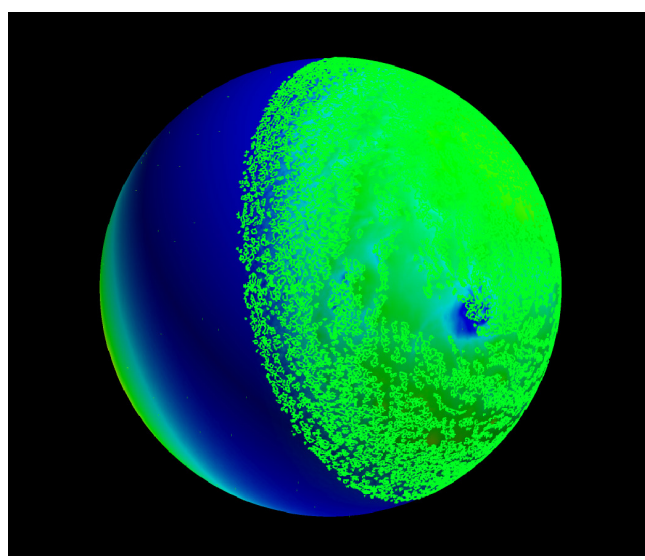

(h) $R e_{p}=1.14 \times 10^{6}, \Gamma=0.2$

Fig. 7. Instantaneous static pressure coefficient on sphere surface and iso-line of the zero-shear stress on sphere surface under various conditions

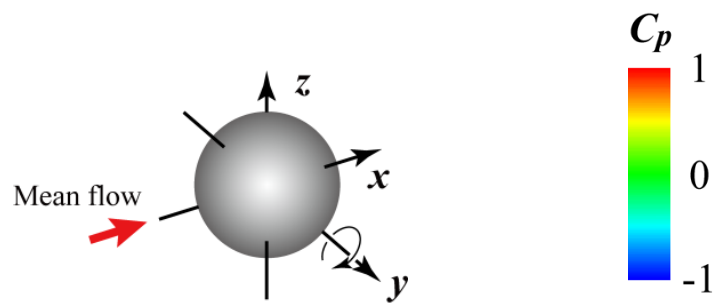

Fig. 8. Coordinate system, rotational direction (left), and color bar corresponding to pressure coefficient $C_{p}$ on sphere surface (right) for Figs. 7(a) to (i)

\section{Summary}

We numerically simulated the flow field around a rotating sphere around a critical Reynolds number by using large-eddy simulation. Unsteady characteristics of the 
boundary layer and a distribution of separation points were visualized at $1.0 \times 10^{4}$, $2.0 \times 10^{5}$, and $1.14 \times 10^{6}$ in the context of boundary-layer transition.

- In the critical flow range at $R e_{p}=2.0 \times 10^{5}$, spatial fluctuation of the velocity field captured as vortical structures, appeared from much upstream on the upstream-moving side than on the downstream-moving side, and the laminar separation points were indistinct. This resulted in a spatially perturbed distribution of the zero-shear separation points on the upstream-moving side and a negative Magnus effect.

- In the subcritical flow range at $R e_{p}=1.0 \times 10^{4}$, in which the surface-boundary layer is subjected to full laminar separation, the zero-shear separation points were evenly distributed on both the upstream- and downstream-moving sides.

- Finally, in the supercritical flow range at $R e_{p}=1.14 \times 10^{6}$, in which the surfaceboundary layer is subjected to fully turbulent separation, the zero-shear separation points were not evenly distributed and did not depend on the sphere rotational speed.

\section{Acknowledgments}

This study was funded by the Ministry of Education, Culture, Sports, Science and Technology, Japan with Grants-in-Aid for Scientific Research (C) 20560143 and 23560179 . We are grateful to Mr. Tadashi Yamazaki of Hokkaido University for his valuable assistance in visualizing the numerical results.

\section{References}

${ }^{1}$ Magnus, G., Über die Abweichung der Geschosse, und: Über eine abfallende Erscheinung bei rotierenden Körpern, (On the deviation of projectiles, and: On a sinking phenomenon among rotating bodies) Poggendorff's Annalen der Physik und Chemie, 164, 1-29 (1853)

${ }^{2}$ Taneda, S., Negative Magnus Effect, Report of Research Institute for Applied Mechanics, 5(20), 123-128 (1957)

${ }^{3}$ Maccoll, J. H., Aerodynamics of a Spinning Sphere, Journal of the Royal Aeronautical Society, 28, 777-798 (1928)

${ }^{4}$ Davies, J. M., The Aerodynamics of Golf Balls, Journal of Applied Physics, 20, 821-828 (1949)

${ }^{5}$ Muto, M., Tsubokura, M. and Oshima, N., Negative Magnus Lift in a Rotating Sphere at around Critical Reynolds Number, submitted to Physics of Fluids

${ }^{6}$ Schlichting, H., Boundary Layer Theory, (1955), McGraw-Hill

${ }^{7}$ Achenbach, E., Experiments on the flow past spheres at very high Reynolds numbers, Journal of Fluid Mechanics, 54(565), 565-575 (1972) 
${ }^{8}$ Wieselsberger, C., Weitere Feststellungen über die Gesetze des Flüssigkeits und Luftwiderstandes, (Other observations on the laws of fluid and air resistance) Physikalische Zeitschrift, 23, 219-224 (1922)

${ }^{9}$ Tsuji, Y., Morikawa, Y. and Mizuno, O., Experimental Measurement of the Magnus Force on a Rotating Sphere at Low Reynolds Numbers, Journal of Fluids Engineering, 107, 484-488 (1985) 